\title{
Ab-Initio Kinetics of Heterogeneous Catalysis: $\mathrm{NO}+\mathrm{N}+\mathrm{O} / \mathrm{Rh}(111)$
}

\author{
A.P.J. Jansen, C.G.M. Hermse, F. Frechard, and J.J. Lukkien \\ Schuit Institute of Catalysis, \\ ST/SKA, Eindhoven University of Technology, \\ P. O. Box 513, \\ 5600 MB Eindhoven, \\ The Netherlands, \\ tgtatj@chem.tue.nl, \\ WWW home page: http://www. catalysis.nl/theory/
}

\begin{abstract}
We show that advances in two fields of computational chemistry, Dynamic Monte Carlo simulations and Density-Functional Theory calculations, are now making it possible to do ab-initio kinetics of realistic surface reactions. We present results of simulations of Temperature- Programmed Desorption experiments of NO reduction to $\mathrm{N}_{2}$ and $\mathrm{O}_{2}$ on the $\mathrm{Rh}(111)$ surface. Kinetic parameters were obtained from DensityFunctional Theory calculations with the Generalized Gradient Approximation, making this one of the first, and up till now the most complex, example of ab-initio kinetics in heterogeneous catalysis. Top, hcp, and fcc sites are all involved and also lateral interactions are necessary to understand the kinetics of this system.
\end{abstract}

\section{Introduction}

Although kinetics plays such an important role in catalysis, its theory has for a long time mainly been restricted to macroscopic rate equations. These implicitly assume a random distribution of adsorbates on a catalyst's surface. Effects of interactions between adsorbates (lateral interactions), reactant segregation, site blocking, and defects have only been described ad hoc. With the advent of Dynamic Monte-Carlo (DMC) simulations, also called Kinetic Monte-Carlo simulations, it has only recently become possible to follow the kinetics of reaction systems on an atomic scale, and thus to study these effects properly.

Two developments have played a major role in the advance of DMC. One is the derivation of a Master Equation (ME) on which DMC can be based, and which has kinetic parameters that can be calculated with ab-initio quantum chemical methods. 1] This ME links the processes on an atomic scale to the macroscopic kinetics. The other is the development of new and the improvement of existing DMC algorithms and ways of modeling reaction systems, [2]34] and the implementation of the algorithms in the general-purpose code CARLOS that allows a user to simulate almost any system of surface reactions. [5] Lateral interactions, steps, and other defects can now easily be included, whereas reactant segregation and site blocking can often be seen in simulations as a consequence of the reaction model. 
DMC simulations need as input the kinetic parameters of the ME. For simulations of real systems these must either be obtained from experiments or be calculated. In many cases it has proved to be very hard to get all parameters from experiments. In particular lateral interactions are difficult to derive from experimental data. Here another computational advance has shown itself to be very important. Lateral interaction, and also other kinetic parameters, may be obtained from quantum chemical calculations. In particular Density-Functional Theory (DFT) with the Generalized Gradient Approximation (GGA) has been shown to give quite accurate results. 6

In this paper we show results for the reduction of $\mathrm{NO}\left(2 \mathrm{NO} \rightarrow \mathrm{N}_{2}+\mathrm{O}_{2}\right)$ on a $\mathrm{Rh}(111)$ surface using DMC simulations with kinetic parameters which are to a large extent calculated with DFT-GGA. This system is important for exhaust gas catalysis. Not only are lateral interactions present in this system, but also three different adsorption sites are involved. We think that this is currently the most complex system for which ab-initio kinetics has been done.

\section{Computational Details}

In this section we briefly discuss the theory of DMC and DFT, and we present the reaction models that we have used.

\subsection{Dynamic Monte Carlo Simulations}

Three parts can be distinguished in our DMC method: the model representing the catalyst and the adsorbates, the ME that describes the evolution of the system, and the DMC algorithms to solve the ME. The ME is given by 17]

$$
\frac{d P_{\alpha}}{d t}=\sum_{\beta}\left[W_{\alpha \beta} P_{\beta}-W_{\beta \alpha} P_{\alpha}\right],
$$

where $\alpha$ and $\beta$ refer to the configuration of the adlayer, the $P$ 's are the probabilities of the configurations, $t$ is real time, and the $W$ 's are transition probabilities per unit time. These transition probabilities give the rates with which reactions change the occupations of the sites. They are very similar to macroscopic reaction rate constants and we will use this term in the rest of this paper, although one should remember that they refer to reactions at the atomic scale. $W_{\alpha \beta}$ corresponds to the reaction that changes $\beta$ into $\alpha$. A configuration is the assignment of the adsorbates to the sites in the system.

The derivation of the ME shows that the rate constants can be written as

$$
W_{\alpha \beta}=\frac{k_{B} T}{h} \frac{Q^{\ddagger}}{Q} \exp \left[-\frac{E_{\mathrm{bar}}}{k_{B} T}\right],
$$

with $k_{B}$ the Boltzmann-constant, $h$ Planck's constant, $T$ temperature, and $E_{\text {bar }}$ the activation barrier of the reaction that transforms configuration $\beta$ into configuration $\alpha$. The partition function $Q^{\ddagger}$ and $Q$ can be interpreted as the partition 
functions of the transition state and the reactants, respectively, although there are some small, generally negligible, differences.[1] The important point is that this equation makes an ab-initio approach to kinetics possible.

The DMC simulations form a powerful numerical method to solve the ME exactly. In fact there are numerous DMC algorithms that might be used: a recent taxonomy of these algorithms contained no less than 48. 4. Most of them are not efficient for any reaction system, however. For a general ME various algorithms have been given by Binder. [8] (Note, however, that here the ME exists before the DMC algorithms, whereas in equilibrium MC it is the other way around. As a consequence $t$ is real time, and not some time in MC steps.) DMC algorithms for rate equations have even been given earlier by Gillespie. 910 For latticegas systems the algorithms given by Binder and Gillespie can be made much more efficient, and also other algorithms can be used. 2344 All DMC algorithms generate an ordered list of times at which a reaction takes place, and for each time in that list the reaction that occurs at that time. A DMC simulation starts with a chosen initial configuration. The list is traversed and changes are made to the configuration corresponding to the occurring reactions. The various algorithms differ in how the reaction times are computed, how a reaction of a particular type is chosen, and how it is determined where on the surface a reaction takes place.

\subsection{Density-Functional Theory Calculations of Kinetic Parameters}

DFT calculations have become very popular for doing quantum chemistry, as DFT forms a good combination of efficiency and accuracy. [6] We have done DFT calculations with the VASP code. 11 12] This code solves the Kohn-Sham equations with a plane wave basis set and the ultrasoft pseudopotentials introduced by Vanderbilt and generated by Kresse and Hafner. [13[14] The Generalized Gradient Approximation of Perdew and Wang has been used (DFT-GGA), because it generally yields good bond energies. 15. The calculations were done with an energy cut-off of $400 \mathrm{eV}$ for the basis, $k$-point sampling of $5 \times 5 \times 1$, and a surface model consisting of a supercell with a slab of five metal layers separated by a vacuum of $13.4 \AA$. The supercells used have a $2 \times 2,3 \times 3$, or a $c(2 \times 4)$ structure and various combinations of NO, N, and O. 16 17 Adsorbates were put on both sides of the slab so that the system was inversion symmetric, avoiding dipoledipole interactions between the slabs. The calculations were converged to within $5 \mathrm{~kJ} . \mathrm{mol}^{-1}$ for the adsorption energy. The calculations allowed us to calculate the lateral interactions for adsorbates on the following combinations of sites: neighboring top sites, neighboring fcc sites, neighboring hcp sites, an fcc site and the next-nearest top site, an fcc site and the next-nearest hcp site, an hcp site and the next-nearest top site, an hcp site and the next-nearest fcc site, and a top site and the next-nearest fcc and hcp sites (see figure 1). The interactions between adsorbates on a top site and the nearest fcc and hcp sites, and between nearest fcc and hcp sites are very strongly repulsive. These interactions have not been calculated, and we have assumed that adsorbates will never occupy these pairs of sites at the same time. 


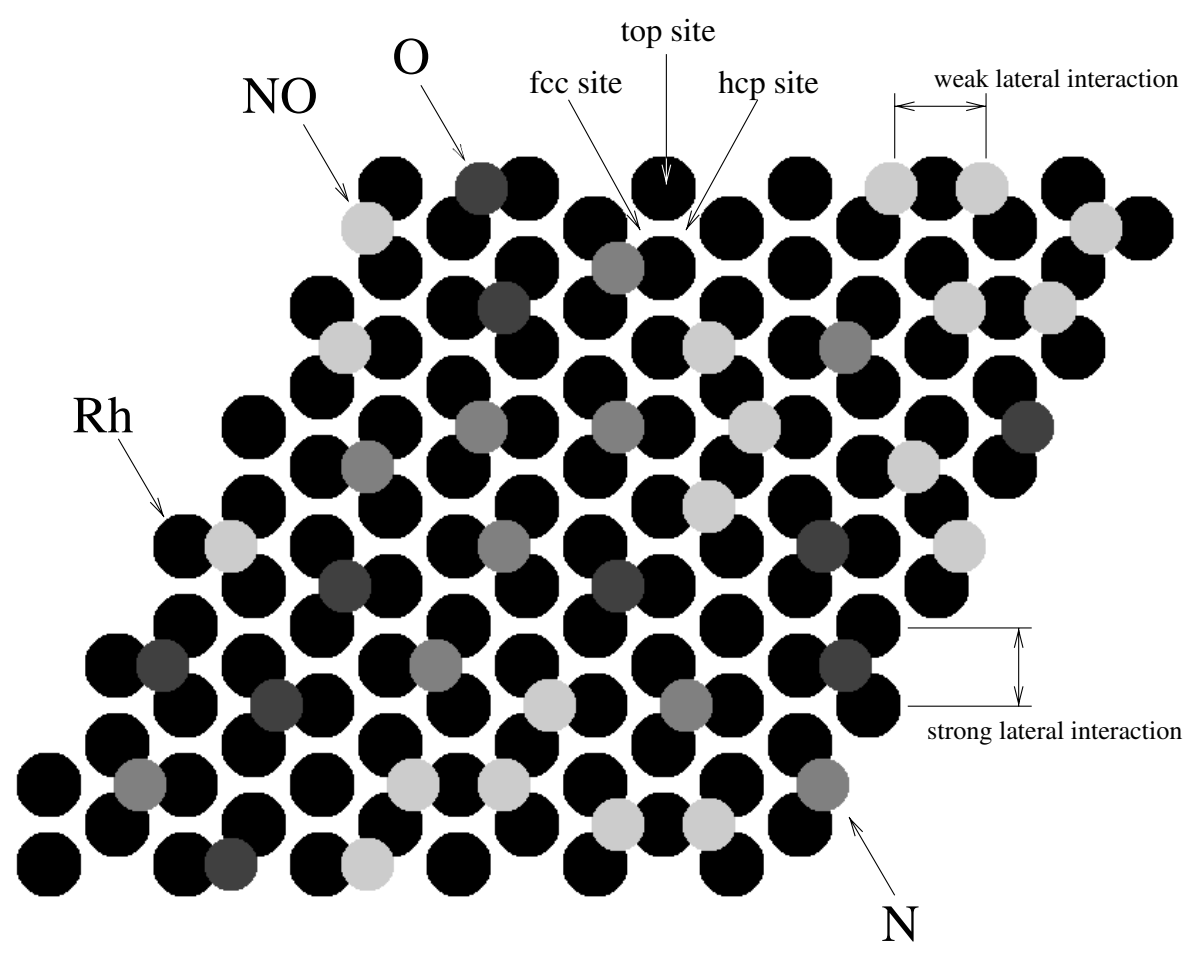

Fig. 1. A snapshot of a small part of the Rh(111) surface with adsorbates during a simulation of the 3 -site model. Only the top $\mathrm{Rh}$ layer and the adlayer are shown. The double arrows indicate the two distances at which lateral interactions work. Lateral interactions between adsorbates that get closer are extremely repulsive, and the simulations do not allow such close approaches. In the 1-site model only hcp sites (or only fcc sites) are used. 


\subsection{The Reaction Models}

NO, atomic nitrogen, and atomic oxygen all prefer threefold adsorption sites (see figure 11). As a first model, the 1-site model, we have assumed that there is only one threefold site (either the fcc or the hcp) relevant for the kinetics. We have simulated the Temperature-Programmed Desorption (TPD) of NO on $\mathrm{Rh}(111)$. In a TPD experiment an adsorbate, in our case NO, is deposited on a catalyst at a low temperature at which no reactions occur. Then the temperature is raised and the rate of desorption is measured. A peak in the desorption rate is generally interpreted as desorption from a particular type of site: the temperature of the peak depends on the bonding energy. Reactions on the surface and lateral interactions can complicate the interpretation of TPD spectra enormously.

The reactions that can occur in our model of the TPD experiment are

$$
\begin{aligned}
\mathrm{NO}(\text { ads })+* & \rightarrow \mathrm{N}(\text { ads })+\mathrm{O}(\text { ads }), \\
\mathrm{NO}(\text { ads }) & \rightarrow \mathrm{NO}(\text { gas })+*, \\
2 \mathrm{~N}(\text { ads }) & \rightarrow \mathrm{N}_{2}(\text { gas })+2 *,
\end{aligned}
$$

where $*$ stands for a vacant site. The two sites involved in all reactions, except for the NO desorption, are nearest neighbors. There is no $\mathrm{O}_{2}$ desorption in our model because this takes place at much higher temperatures than the other reactions. NO dissociation is suppressed at high coverages, because of site blocking: i.e., it needs a neighboring vacant site, which may not be present. There is an additional suppression due to lateral interaction, which also influence the other reactions. We have included lateral interactions through the Polanyi-Brønsted relation for the activation barrier: 18

$$
E_{\mathrm{bar}}=E_{\mathrm{bar}}^{(0)}+\alpha\left(\Delta \Phi_{\text {product }}-\Delta \Phi_{\text {reactant }}\right),
$$

were $E_{\mathrm{bar}}^{(0)}$ is the activation barrier without lateral interactions, and $\Delta \Phi_{\text {product }}$ $\left(\Delta \Phi_{\text {reactant }}\right)$ is the change in adsorption energy of the products (reactants) due to lateral interactions. The coefficient $\alpha$ is the Brønsted-coefficient. It was taken equal to 1 for all reactions (late barrier) except for diffusion for which we used $\alpha=1 / 2$. We have assumed that the lateral interactions are pairwise additive: $\Delta \Phi_{\text {product }}$ and $\Delta \Phi_{\text {reactant }}$ are simply the sums of pair interactions. DFT calculations gave no indications that this is incorrect. Only lateral interactions with nearest neighbors were included in the 1-site model. Using realistic diffusion rate constants would make the simulations too costly. The rate constants that we have used (see table 11) are five to eight orders of magnitude smaller than the real ones. However the changes in the results when the rate constants for diffusion are increased are negligible.

In the 3-site model we included both threefold sites (fcc and hcp) and the onefold (top) site in the unit cell. The reason for this more complex model was not based on the kinetic results of the simulations with the 1-site model, but on the discrepancy for the lateral interactions between the DFT calculations and fitted values with the 1-site model (see below). Also a LEED study of $\mathrm{NO} / \mathrm{Rh}(111)$ 
Table 1. Activation energies and prefactors for the reactions in the 3-site model when there are no lateral interactions. Nitrogen and oxygen diffuse only between 3 -fold sites.

$\begin{array}{ccc}\text { reaction } & \begin{array}{c}\text { prefactor } \\ \text { (in } 1 / \mathrm{sec})\end{array} & \begin{array}{c}\text { activation energy } \\ \text { (in } \mathrm{kJ} / \mathrm{mol} \text { ) }\end{array} \\ \text { NO dissociation } & 10^{11} & 65 \\ \text { NO desorption (3-fold) } & 10^{13.5} & 99 \\ \text { NO desorption (top) } & 10^{13.5} & 52 \\ \mathrm{~N}_{2} \text { desorption } & 10^{10} & 120 \\ \text { NO diffusion }(3 \text {-fold } \rightarrow 3 \text {-fold) } & 10^{5} & 22.5 \\ \text { NO diffusion (3-fold } \rightarrow \text { top) } & 10^{5} & 47 \\ \text { NO diffusion (top } \rightarrow 3 \text {-fold) } & 10^{5} & 0 \\ \text { N diffusion } & 10^{5} & 16 \\ \text { O diffusion } & 10^{8} & 45\end{array}$

with high coverage indicates that NO can be distributed over three sites in our 3-site model.[19] The reactions are the same as for the 1-site model, but, as we have more sites, we have more different lateral interactions.

\section{Results and Discussion}

The results of the DMC simulations obtained with the 1-site model are in reasonable agreement with the experiment. The kinetic parameters are obtained by fitting to the experimental data. For some parameters this is quite straightforward. For example, the activation energy and the prefactor for $\mathrm{N}_{2}$ desorption can be obtained from a TPD experiment starting with a low coverage layer of nitrogen that has been atomically deposited. 20 The low coverage is necessary to avoid effects of lateral interactions. Similarly the activation energies and prefactors for NO dissociation can be obtained. It's much harder to obtain kinetic parameters for the NO desorption, because this only occurs when many other adsorbates are present. These adsorbates block the sites necessary for the NO dissociation which would otherwise occur. Their lateral interactions influence, however, the NO desorption. It is also very hard to obtain the lateral interactions themselves from experiments. We have determined these parameters by varying them, by hand, and by fitting the simulated TPD spectra to the experimental ones. There are two problems with the kinetic parameters for the 1-site model. First the TPD experiments do not seem to contain enough data to determine the lateral interactions uniquely. Various sets of them were obtained by different people which gave fits of similar quality. Second, there is a huge discrepancy with DFT results: DFT gives values for the same lateral interactions that are almost an order of magnitude larger than what is obtained from fitting to the TPD spectra. Using the DFT results in the 1-site model gave totally incorrect spectra.

We have extended the 1 -site model to the 3 -site model to resolve the discrepancy between the fitted and the calculated lateral interactions (see figure 2). The main difference in the structures that the adlayer forms in the simulations 


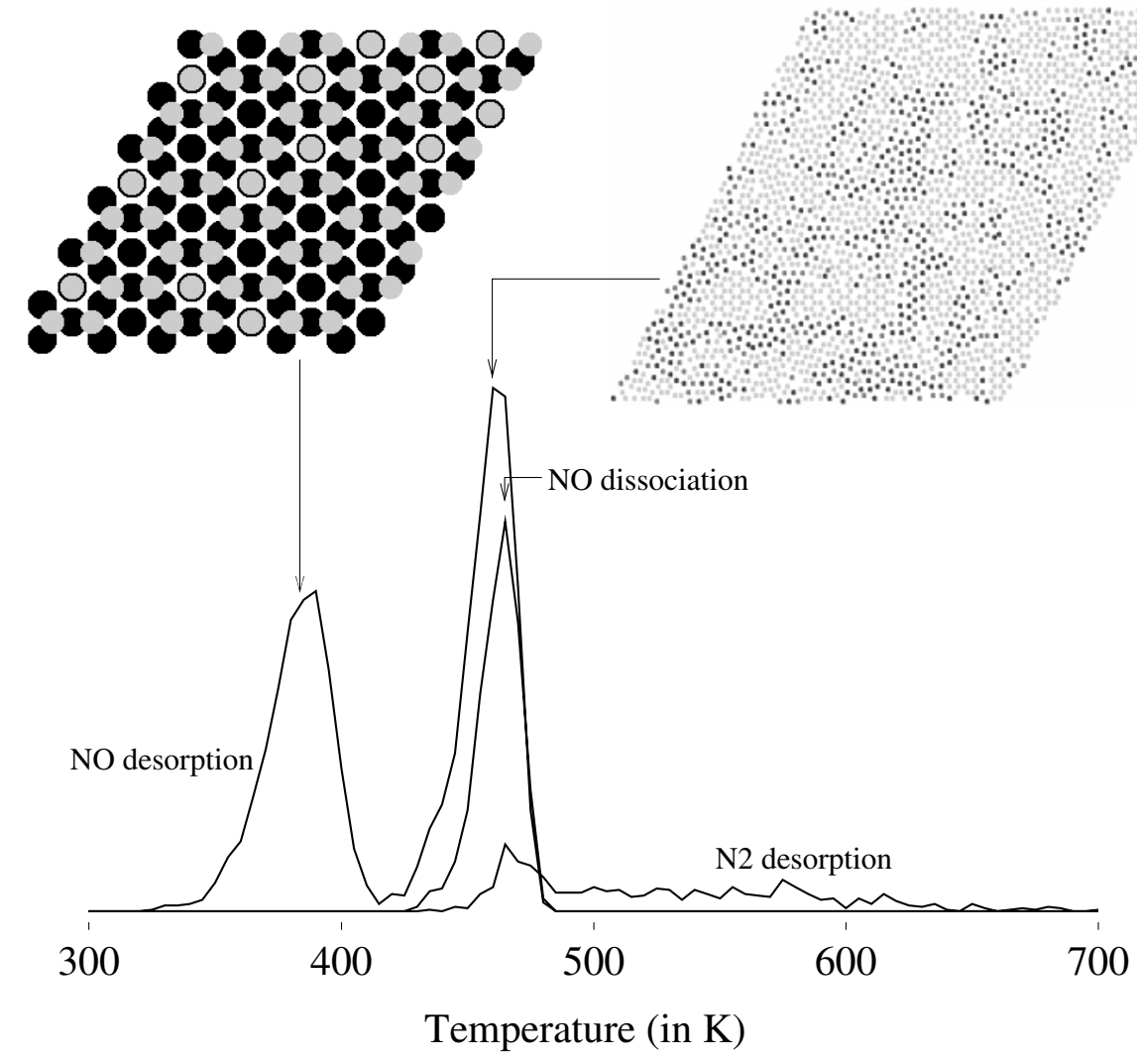

Fig. 2. Snapshots and reaction rates as a function of temperature from a simulation of the TPD experiment with a maximum initial NO coverage $\left(\theta_{\mathrm{NO}}=0.75\right)$ and a heating rate of $10 \mathrm{~K} . \mathrm{s}^{-1}$. The 3 -site model was used. The desorptions can be measured by TPD, but another technique is necessary to measure the NO dissociation. The top-left snapshot shows the top Rh layer and the adsorbates (only NO) of a small part of the system at $T=385 \mathrm{~K}$. The top-right snapshot shows only the adsorbates of the whole system at $T=458 \mathrm{~K}$. 
with the 1 - and the 3 -site model is that in the 3 -site model the adsorbates can stay farther apart at high coverages. Consequently the system avoids almost completely lateral interactions for which the DFT calculations give high values. Occasionally adsorbates do get as close to each other as in the 1-site model, but only when it's necessary for restructuring the adlayer. These high lateral interactions do not show up in the TPD spectra. The lateral interactions and other kinetic parameters are given in tables 1 and 2

Table 2. Lateral interactions (in $\mathrm{kJ} / \mathrm{mol}$ ) in the 3 -site model for two adsorbates at distances 1 and $2 / \sqrt{3}$ times the distance between two Rh atoms. (Interactions between two NO molecules both at top sites have not been calculated because close NO at top sites doe not occur due to the low adsorption energy at top sites.)

\begin{tabular}{|c|c|c|c|c|c|c|c|}
\hline \multirow[b]{2}{*}{ NO (3-fold) } & \multicolumn{2}{|c|}{$\begin{array}{c}\text { NO } \\
\text { (3-fold) }\end{array}$} & $\begin{array}{c}\mathrm{NO} \\
\text { (top) }\end{array}$ & \multicolumn{2}{|c|}{$\begin{array}{c}\mathrm{N} \\
\text { (3-fold) }\end{array}$} & \multicolumn{2}{|c|}{$\begin{array}{c}\mathrm{O} \\
(3 \text {-fold })\end{array}$} \\
\hline & 26 & 0 & - & 23.5 & 16 & 101 & 15 \\
\hline $\mathrm{NO}$ (top) & & & & - & 0 & - & 6.5 \\
\hline N (3-fold) & & & & 40 & 21.5 & 45.5 & 25 \\
\hline O (3-fold) & & & & & & 26 & 26 \\
\hline
\end{tabular}

The maximum coverage of NO (i.e., the maximum number of NO molecules on the surface per Rh atom in the top layer of the catalyst) is 0.75 . The partial occupation of sites of the same type indicates that there are repulsive lateral interactions. In the 3 -site model the coverage $\theta_{\mathrm{NO}}=0.75$ corresponds to the $(2 \times 2)-3 \mathrm{NO}$ structure in which a quarter of each of the top, hcp, and fcc sites are occupied. In this structure the NO molecules feel only the weak repulsive interaction. Putting more NO molecules on the catalyst immediately leads to strong repulsive interactions (see figure 1). In the 1-site model there is only a gradual increase in the lateral interaction energy with coverage, and it's not clear in this model why the maximum coverage should be 0.75 .

Dissociation of isolated NO is a fast reaction. As the dissociation products, $\mathrm{N}$ and $\mathrm{O}$, need one extra site, the dissociation will not occur, however, when sites are blocked or when lateral interactions increase the activation barrier too much. In that case NO dissociation will only occur after the slower NO desorption has made sufficient room on the catalyst's surface. Figure 2 shows that the NO molecules at the top sites desorb first. They are bound less strongly to the surface. This does not lead to sites useful for NO dissociation yet. Only when NO molecules vacate hcp and fcc sites NO dissociation begins.

At a slightly higher temperature $\mathrm{N}_{2}$ desorption also starts taking place. The $\mathrm{N}_{2}$ desorption takes place over a very broad temperature range and the peak at the beginning of that range is also caused by lateral interactions. The difference in lateral interactions between $\mathrm{NO}, \mathrm{N}$, and $\mathrm{O}$ causes segregation into $\mathrm{NO}$ and $\mathrm{N}+\mathrm{O}$ islands. $\mathrm{O}_{2}$ desorption only occurs at much higher temperatures and is not shown in figure 2 . 


\section{Conclusions}

We have done DMC simulations of the NO reduction to $\mathrm{N}_{2}$ and $\mathrm{O}_{2}$ on $\mathrm{Rh}(111)$ with kinetic parameters obtained from DFT calculations. Our 3-site model has three different sites per unit cell, site blocking, and lateral interactions for all reactions and for diffusion. The DMC simulations gave results for real time dependence and used exact reaction rate constants for simulating TPD spectra. The DFT calculations were done with a plane wave basis set and ultrasoft pseudo-potentials on a five-layer slab and supercells up to $3 \times 3$ structures.

The present study shows the necessity of DMC simulations with DFT calculations to understand kinetics in heterogeneous catalysis. A 1-site model shows that it's well possible to reproduce the experimental kinetics, but that this does not mean that the model is correct. Only the comparison between fitted and calculated kinetic parameters shows the shortcomings of this too simple model. The results of the 3-site model with kinetic parameters obtained from DFT calculations indicates that it is becoming possible to do ab-initio kinetics for real-world applications.

\section{References}

1. Gelten, R. J., van Santen, R. A., and Jansen, A. P. J.: Dynamic Monte Carlo simulations of oscillatory heterogeneous catalytic reactions. In P. B. Balbuena and J. M. Seminario, editors, Molecular Dynamics: From Classical to Quantum Methods. Elsevier, Amsterdam (1999)

2. Jansen, A. P. J.: Monte Carlo simulations of chemical reactions on a surface with time-dependent reaction-rate constants. Comput. Phys. Comm. 86, (1995) 1-12

3. Lukkien, J. J., Segers, J. P. L., Hilbers, P. A. J., Gelten, R. J., and Jansen, A. P. J.: Efficient Monte Carlo methods for the simulation of catalytic surface reactions. Phys. Rev. E 58, (1998) 2598-2610

4. Segers, J. P. L.: Algorithms for the Simulation of Surface Processes. Ph.D. thesis, Eindhoven University of Technology (1999)

5. Carlos is a general-purpose program, written in C by J. J. Lukkien, for simulating reactions on surfaces that can be represented by regular grids; an implementation of the first-reaction method, the variable stepsize method, and the random selection method. Write to tgtatj@chem.tue.nl if you are interested in using Carlos.

6. Koch, W. and Holthausen, M. C.: A Chemist's Guide to Density Functional Theory. Wiley-VCH, New York (2000)

7. van Kampen, N. G.: Stochastic Processes in Physics and Chemistry. NorthHolland, Amsterdam (1981)

8. Binder, K.: Monte Carlo Methods in Statistical Physics. Springer, Berlin (1986)

9. Gillespie, D. T.: A general method for numerically simulating the stochastic time evolution of coupled chemical reactions. J. Comput. Phys. 22, (1976) 403-434

10. Gillespie, D. T.: Exact stochastic simulations of coupled chemical reactions. J. Phys. Chem. 81, (1977) 2340-2361

11. Kresse, G. and Furthmüller, J.: Efficiency of ab-initio total energy calculations for metals and semiconductors using a plane-wave basis set. Comput. Mat. Sci. 6, (1996) 15-50 
12. Kresse, G. and Furthmüller, J.: Efficient iterative schemes for ab initio total-energy calculations using a plane-wave basis set. Phys. Rev. B 54, (1996) 11169-11186

13. Vanderbilt, D.: Soft self-consistent pseudopotentials in a generalized eigenvalue formalism. Phys. Rev. B 41, (1990) 7892-7895

14. Kresse, G. and Hafner, J.: Norm-conserving and ultrasoft pseudopotentials for first-row and transition elements. J. Phys.: Condens. Matter 6, (1994) 8245-8257

15. Perdew, J. P.: Unified theory of exchange and correlation beyond the local density approximation. In P. Ziesche and H. Eschrig, editors, Electronic Structure of Solids '91, 11. Akademie Verlag, Berlin (1991)

16. Loffreda, D., Simon, D., and Sautet, P.: Molecular and dissociative chemisorption of NO on palladium and rhodium (100) and (111) surfaces: A density-functional periodic study. J. Chem. Phys. 108, (1998) 6447-6457

17. Thomas, J. M. and Thomas, W. J.: Principles and Practice of Heterogeneous Catalysis. VCH, Weinheim (1997)

18. van Santen, R. A. and Niemantsverdriet, J. W.: Chemical Kinetics and Catalysis. Plenum Press, New York (1995)

19. Zasada, I., Hove, M. A. V., and Somorjai, G. A.: Reanalysis of the Rh(111) $+(2 \times$ 2) - 3NO structure using automated tensor leed. Surf. Sci. 418, (1998) L89-L93

20. van Hardeveld, R. M., van Santen, R. A., and Niemantsverdriet, J. W.: Formation of $\mathrm{NH}_{3}$ and $\mathrm{N}_{2}$ from atomic nitrogen, and hydrogen on rhodium(111). J. Vac. Sci. Technol. A 15, (1997) 1558-1562 